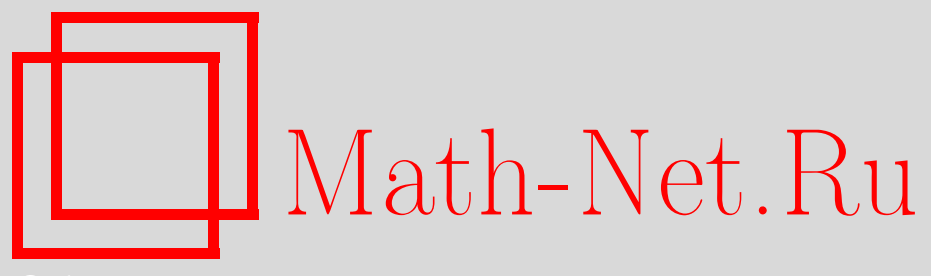

В. М. Максимов, Вероятностные структуры и вероятностные множества, УМН, 2007, том 62, выпуск 6, 181182

DOI: https://doi.org/10.4213/rm7597

Использование Общероссийского математического портала Math-Net.Ru подразумевает, что вы прочитали и согласны с пользовательским соглашением http://www .mathnet.ru/rus/agreement

Параметры загрузки:

IP : 54.198 .187 .58

26 апреля 2023 г., 15:35:07

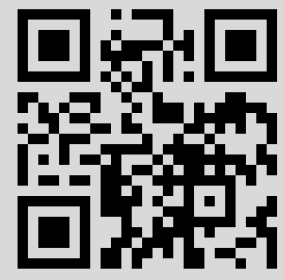




\section{Вероятностные структуры и вероятностные множества}

\section{В. М. Максимов}

В последние 60 лет постоянно появляются исследования, в которых переосмысливаются и расширяются понятия случайности и вероятности [1]-[6]. Так, А. Н. Колмогоров предложил связать понятие случайности объекта с алгоритмической сложностью его построения [1], см. также [2]. Другим важным аспектом случайности является ее "количественная" оценка. Начиная с П. Дирака [3] в физике стали рассматривать отрицательные и комплексные вероятности [4]. Позже в [5] была построена $p$-адическая квантовая механика, а в [6] - p-адическая теория вероятностей. В связи с этим в [7], [8] автором были введены алгебраические структуры, элементами которых можно оценивать меру случайности.

ОПределение 1. Множество $\mathscr{P}$ называется вероятностным множеством, если в нем определены бинарная операция умножения с единицей 1 и неполная бинарная операция сложения, в которой есть нулевой элемент $\mathbf{0}$, такие, что эти операции коммутативны, ассоциативны, дистрибутивны и удовлетворяют условиям:

1) для любого $\alpha \in \mathscr{P}$ существует дополнительный элемент $\bar{\alpha}, \alpha+\bar{\alpha}=\mathbf{1}$;

$2)$ для любого ненулевого элемента $\alpha \in \mathscr{P}$ сумма $\alpha+\cdots+\alpha$ ( $k$-раз) не определена начиная с некоторого $k=k(\alpha)$;

3) уравнение $x^{2}=x$ в $\mathscr{P}$ имеет только два решения $\mathbf{0}$ и $\mathbf{1}$ (это условие мы называем условием Колмогорова).

Например, множество чисел, принадлежащих отрезку $[0,1]$, является вероятностным множеством относительно обычного сложения и умножения.

Свойства 1)-3) позволяют строить $\mathscr{P}$-меры и случайные величины с $\mathscr{P}$-распределением, сохраняя при этом естественные представления о вероятности, такие как свойство неразложимости нуля $(\alpha+\beta=\mathbf{0} \Rightarrow \alpha=\beta=\mathbf{0})$ и свойство недополняемости до единицы, т. е. сумма $\mathbf{1}+\alpha$ не определена в $\mathscr{P}$ для $\alpha \neq \mathbf{0}$. Кроме того, свойство 2) позволяет показать (см. [8]), что если $\alpha \neq \mathbf{0}$, то и $\alpha^{2} \neq \mathbf{0}$, а свойство 3) обеспечивает выполнение закона нуля или единицы (см. [9]) для сумм независимых случайных величин с $\mathscr{P}$-распределением.

ОПРеДЕЛЕниЕ 2. Множество $\mathbb{P}$ называется вероятностной структурой, если в нем определены бинарная операция умножения с единицей 1 и неполная бинарная операция сложения, в которой есть нулевой элемент $\mathbf{0}$, такие, что эти операции коммутативны, ассоциативны, дистрибутивны и удовлетворяют условиям:

1) для любого $\alpha \in \mathbb{P}$ существует дополнительный элемент $\bar{\alpha}, \alpha+\bar{\alpha}=\mathbf{1}$;

$2 \mathrm{a})$ для любого ненулевого элемента $\alpha \in \mathbb{P}$ сумма $1+\alpha$ не определена в $\mathbb{P}$.

В вероятностной структуре также имеет место неразложимость нуля; действительно, если $\alpha+\beta=\mathbf{0}$, то, прибавляя к обеим частям $\bar{\beta}$, получим $(\alpha+\beta)+\bar{\beta}=\alpha+(\beta+\bar{\beta})=$ $\alpha+\mathbf{1}=\bar{\beta}$, что противоречит свойству $2 \mathrm{a})$, если $\alpha \neq \mathbf{0}$. Следовательно, $\alpha=\beta=\mathbf{0}$.

Нетрудно показать (см. [8]), что из 2) следует 2a), т. е. каждое вероятностное множество является и вероятностной структурой. Однако вероятностная структура может не удовлетворять как условию 2), так и условию 3 ).

Теорема 1. Существуют вероятностные структуры с условием Колмогорова, которые не являются вероятностным множеством

ДоказАтельство. Рассмотрим множество $\mathscr{F}$, состоящее из всех конечных сумм полиномов вида $x^{v_{0}}(1-x)^{v_{1}} \cdots(1-n x)^{v_{n}}, v_{i}=0,1,2, \ldots, n=0,1,2, \ldots$, от действительного переменного $x$ и из функции $\mathbf{0}=0(x) \equiv 0$. Множество $\mathscr{F}$ содержит тождественную единицу $\mathbf{1}=1(x) \equiv 1$, и каждый образующий полином имеет 
в $\mathscr{F}$ дополнительный до 1 элемент, который равен $P(x)+x^{v_{0}} \cdots(1-n x)^{v_{n}-1} n x$, где $P(x)$ есть дополнительный элемент для образующего полинома $x^{v_{0}}(1-x)^{v_{1}} \cdots(1-$ $(n-1) x)^{v_{n-1}}(1-n x)^{v_{n}-1}$ в $\mathscr{F}$. Следовательно, $P(x)$ находится по индукции. Теперь рассмотрим множество $\mathbb{P}$ тех элементов из $\mathscr{F}$, которые можно дополнить до единицы $\mathbf{1}=1(x)$ в $\mathscr{F}$. Умножение и сложение в $\mathbb{P}$ наследуются из $\mathscr{F}$. Сумма $1+\alpha(x)$ для ненулевого $\alpha$ в $\mathbb{P}$ не определена. Действительно, если допустить, что $1+\alpha(x)=\beta(x) \in \mathbb{P}$, то, прибавляя к обеим частям $\bar{\beta}(x)$ и учитывая ассоциативность, получим $(\mathbf{1}+\alpha(x))+\bar{\beta}(x)=\mathbf{1}+\gamma(x)=\mathbf{1}$. Очевидно, что каждый элемент $f(x)$ из $\mathscr{F}$ в некоторой окрестности интервала $\left(0, \varepsilon_{f}\right), \varepsilon_{f}>0$, больше нуля, что противоречит равенству $\mathbf{1}+\gamma(x)=\mathbf{1}$. Итак, $\gamma(x)=\mathbf{0}$. По тем же соображениям имеем $\alpha(x)=\mathbf{0}$. Следовательно, $\mathbb{P}$ есть вероятностная структура. С другой стороны, для элемента $\alpha=x$ сумма $\alpha+\cdots+\alpha=n x$ определена в $\mathbb{P}$ вместе с $1-n x$ для любого $n$. Следовательно, $\mathbb{P}$ не обладает свойством 2). Из $f^{2}=f \in \mathscr{F}$ следует, что $f(x) \equiv 1$ или $f(x) \equiv 0$. Теорема 1 доказана.

Известно, что с каждым булеаном (т. е. множеством подмножеств некоторого множества $M)$ связаны ассоциативная решетка и булева алгебра. Аналогично, с каждым булеаном $\mathscr{B}$ можно связать и вероятностную структуру $\mathbb{P}(\mathscr{B})$. Элементами $\mathbb{P}(\mathscr{B})$ являются множества из $\mathscr{B}$ с умножением $A \cdot B=A \cap B$ и сложением $A+B=A \cup B$, которое определено, только если $A \cap B=\varnothing$. При этом $\mathbf{0}=\varnothing, \mathbf{1}=M$ и $\bar{A}=M \backslash A$. Если $\mathscr{B}$ содержит более двух элементов, то $\mathbb{P}(\mathscr{B})$ не является вероятностным множеством, так как $A^{2}=A$ для любого $A \in \mathscr{B}$.

Теорема 2. Каждая конечная вероятностная структура $\mathbb{P}$ является вероятностной структурой конечного булеана.

Идея доказательства состоит в следующем. Из конечности вероятностной структуры $\mathbb{P}$ выводится разложение единицы: $\mathbf{1}=e_{1}+\cdots+e_{s}$, где $e_{i}$ - ортогональные идемпотенты, т. е. $e_{i} e_{j}=\mathbf{0}, e_{i}^{2}=e_{i}$. Элементами $\mathbb{P}(\mathscr{B})$ будут все суммы $e_{i_{1}}+\cdots+e_{i_{k}}$, $1 \leqslant i_{1}<\cdots<i_{k} \leqslant s$, а соответствующий булеан $\mathscr{B}$ состоит из подмножеств множества $\left\{e_{1}, \ldots, e_{s}\right\}$.

Таким образом, всякое конечное вероятностное множество состоит из двух элементов 0 и 1.

Что касается компактного вероятностного множества, то весьма правдоподобна гипотеза о том, что оно изоморфно отрезку $[0,1]$ с естественными операциями и топологией.

Автор выражает благодарность О.В. Вискову, Э. Л. Пресману, А. М. Стёпину и А. Ю. Хренникову за внимание к работе и полезные замечания.

\section{Список литературы}

[1] А.Н. Колмогоров, Избранные труды, Т. 3, Теория информации и теория алгоритмов, Наука, M., 2005. [2] R. L. Solomonoff, Information and Control, 7:1 (1964), 1-22. [3] P. A. M. Dirac, Rev. Modern Phys., 17:2-3 (1945), 195-199. [4] R. P. Feynman, Quantum implications, Routledge and Kegan Paul, London, 1987, 235-248. [5] В. С. Владимиров, И. В. Волович, Е.И. Зеленов, р-адический анализ и математическая физика, Наука, М., 1994. [6] А. Ю. Хренников, Неархимедов анализ и его приложения, Физматлит, М., 2003. [7] V. M. Maximov, Foundations of probability and physics, QP-PQ: Quantum Probab. White Noise Anal., 13, World Sci. Publ., River Edge, NJ, 2001, 257-273. [8] V. M. Maximov, Foundations of probability and physics - 4, AIP Conf. Proc., 889, eds. G. Adenier, C. Fuchs, A. Yu. Khrennikov, Amer. Inst. Phys., Melville, NY, 2007, 353-361. [9] А. Н. Колмогоров, Основные понятия теории вероятностей, Наука, М., 1974.

\section{В. M. Максимов (V.M. Maksimov)}

Российский государственный гуманитарный университет; Тверской государственный университет

E-mail: vm_maximov@mail.ru
Представлено А. В. Булинским Принято редколлегией 31.07.2007 